\title{
A Survey of HISs with Information Systems Success Model
}

\author{
${ }^{1}$ Ngqwala and ${ }^{2}$ Van Dyk \\ ${ }^{1,2}$ Faculty of Pharmacy, Rhodes University, Makhanda, 6139, South Africa. \\ 2dyk336@outlook.com
}

\author{
Article Info \\ Journal of Biomedical and Sustainable Healthcare Applications (http://anapub.co.ke/journals/jbsha/jbsha.html) \\ Doi: https://doi.org/10.53759/0088/JBSHA202202005 \\ Received 26 January 2021; Revised form 20 March 2021; Accepted 25 July 2021. \\ Available online 05 January 2022. \\ (C2022 Published by AnaPub Publications.
}

\begin{abstract}
Hospital Information System (HIS) is a form of healthcare information system that is globalized and applied in the medical sector. Researchers, doctors, and management are all interested in the rate of success of HISs; therefore it's a continuous study topic. At this research, we created a new tool to assess the success rate of HIS in a medical center based on the perspectives of us ers. The research was place in Ebnesina and Mashhad, Persia, at the Dr. Hejazi Mental Center and Educational Facility. A self-administered standardized questionnaire based on Information Systems Success Model (ISSM) was used to gather data, and it included seven factors: systems quality, data quality, quality of service, system use, applicability, fulfillment, and positive externalities. An advisory group checked the content's legitimacy. Cronbach alpha was used to test the consistency and stability of dimensions. To examine the importance of relationships between variables, Correlation and regression was determined. On the basis of user feedback, the HIS rate of success has been established. The research included a approximately 125 participants. A content validity index (CVI) of 0.8 and a validity ratio (CVR) of 0.86 were used by an advisory committee to verify the item. The instruments have an overall Cronbach's alpha of 0.9 . Between the analyzed dimensions, the Pearson's correlation coefficient revealed substantial positive connections. In the institution under investigation, the HIS rate of success averaged 65 percent. (CI: 64 percent, 67 percent). The greatest success rates were found in the aspects of "effectiveness," "systems quality," and "positive externalities." Future research might employ the tool used in this research to evaluate HIS. In this research, a technique for calculating the HIS rate of success depending on user feedback was established. This strategy enables institutional HIS chances of success to be compared. Our results also highlight the perspectives of HIS clients in a developing economy.
\end{abstract}

Keywords - Health Information Systems (HISs), Electronic Health Records (EHRs), Information Systems Success Model (ISSM).

\section{INTRODUCTION}

Health Information Systems (HISs) alludes to the systems formulates to effectively management medical datasets. This integrates the systems that gather, store, manage and transfer Electronic Health Records (EHRs), a clinics' operational management or the healthcare systems supporting medical policy judgments. HISs also integrates the systems, which deal with data about the services of healthcare providers and facilities. Integrated efforts could be leveraged to enhance the results of patients, inform literatures, and influence the process of policy-making and enhance the decision-making process. Since HISs majorly retrieve, procedures, or maintain massive volumes of sensitive datasets; and security is a major concern.

HISs has been introduced in hospitals to offer fast and reliable data, hence meeting administrative demands and enhancing medical efficacy and productivity at an affordable rate. HISs is among the most widely used Health Information Systems. The integrity of these systems is a key source of worry. As a result, it is critical to assess HISs on a regular basis. In addition, HIS deployment requires a significant financial commitment. The HITECH (Hospital Information Technology of Economic and Clinical Health) Act of 2009, for example, secures approximately USD 10 million for every facility in America. As a result, post-implementation evaluations of HIS performance are crucial [1]. The study of the HIS positive outcome is continuing since the consequences are of concern to not only researchers but also doctors and management. The evaluation's findings may demonstrate the usefulness of HIS and give crucial data for future choices.

The Information Systems Success Model (ISSM) (also known as the Delone and McLean Information System success model) is a theory for Information Systems (IS) that focuses on detailed comprehension of IS success based on the identification, description and explanation of the connection between seven of the most fundamental dimension of success alongside the IS are typically assessed. Previous advancements of the theory was considered by Ephraim McLean and William DeLone in 1992 and later modified a decade later based on the responses obtained from other experts researching on the safe segment of research [2]. The success model of IS has been reviewed in many past literature sources regarding HISs.

There are several paradigms for evaluating healthcare data systems now available. The Information Systems Success Model (ISSM) is a well-known and reliable approach for evaluating these technologies. Technology readiness, facilitating conditions, service quality, system usage, effectiveness, fulfilment, and overall benefits are among the seven aspects of the 
ISSM. ISSM specializes in evaluating technical features of data systems from the perspective of users and analyzes the impact of innovation on system usage and contentment. ISSM identifies and defines the connections between seven fundmaental dimensions of ISSM: systems quality, data quality, quality of service, system use, applicability, fulfillment, and positive externalities

- Systems quality: The general quality of systems, like quality of information, is among the most frequent characteristics upon which IS are assessed. By mediational interactions such as use intents and users ' satisfaction components, system quality indirectly influences the amount to which the service is capable of offering advantages.

- Data quality: Data quality alludes to the information quality, which systems are capable of storing, deliverying, and producing, and these are the most common dimensionalities alongside which IS affects both the satisfaction of users with systems and their intentions to utilize systems that affect the dimension to which systems are capable of yielding the merits of users and the firm.

- Quality of service: IS are often assessed in terms of the service quality they can provide, in addition to data and system reliability. Services quality has a significant effect influence on users' intents to employ the systems and their contentment with it, which in turn influenced on the service's overall advantages.

- System use: In the context of IS, the terms "primary intent to utilize an information management system" and "effective system usage" are well-defined concepts. Data, systems, and quality of service are all factors in the ISSM that impact framework use and goals. Systems use is thought to have an impact on a user's happiness with the IS, which in turn has an impact on use expectations. System utilization has a direct impact on the net advantages that the systems may deliver, along with user happiness.

- Applicability: "Effective system utilization" and "main purpose to use an information management system" are well-defined notions. In the ISSM, data, systems, and service quality are all aspects that influence framework utilization and objectives. The usage of systems is assumed to influence a user's satisfaction with the IS, which in turn influences use expectations.

- Fulfillment: Data, systems, and quality of service are all factors that affect the end user. Customer satisfaction, like real system usage, has a direct impact on the data service's net advantages. The degree from which a client is satisfied or satisfied with an IS is referred to as fulfillment, and it is thought to be influenced directly by system usage.

- Positive externalities: An essential aspect of the total value of a data systems to its clients or the underpinning business is the net economic benefit that it may provide. The utilization of the systems and client engagement with the systems influence net systems advantages in the ISSM. System advantages are thought to have an impact on both client happiness and the likelihood of a user using the service.

The interplay of multiple ISSM aspects is critical to the effectiveness of data systems. The Persiaian Department of Medical and Health Training ordered public hospitals to transmit patients' health records to SEPAS (Persia's Health Automated Record Programme) in 2014 [3]. As a result, the use of HISs in clinics throughout the nation has increased dramatically. The success of Health Electronic Records (EHRs) and health information systems (HISs) was assessed after their application in Taiwan, Korea, and Saudi Arabia. As per our understanding, there have been relatively few investigations that have examined the effectiveness of HISs in Persia as an emerging nation. Nonetheless, these trials did not give solid data on the effectiveness of HISs when taken together. As a result, further research is needed to obtain more information concerning the effectiveness and usefulness of HISs in Persia.

In this research, we focus on instrumental development to assess the rate of success of HISs with respect to ISSM that could be applied in comparison to the success rate of HIS in various medical settings. In addition, we review the HISs' success rate with respect of the viewpoint of users in a medical training hospital in Persia. With that regard, this paper has been organized as follows: Section II focuses on the methodology for the research. Section III focuses on the results of the research while Section IV presents the discussions. Lastly, Section V concludes the paper.

\section{METHODOLOGY}

In this research, the approach of descriptive cross-sectional has been considered.

\section{Instruments}

To gather information, a self-administered survey approach centred on ISSM was created. Technology readiness, quality of information, service levels, system usage, effectiveness, contentment, and overall benefits were among the seven aspects of the survey. There are numerous assessment techniques for each category. The questions were created using the ISSM assessment criteria and attributes as a guideline. An experienced team checked the text for accuracy. Nine healthcare information specialists were on the group. A Content Validity Index (CVI) and a Confirmatory Factor Proportion were used to assess expert validity (CVR). In this paper, the Cronbach's alpha was applied in determination of the internalized dependability of each measurement. The importance of relationships between variables was determined using Correlational research design. The object's final document has two parts. Users' attributes such as years old, ethnicity, educational status, monthly work schedule, ICDL qualification, job experience, HIS expertise and computing knowledge ( $\mathrm{n}$ equals to eight questions) are integrated in the first segment. The second segment integrates sixty questions on the five- 
point Likert scale that ranges from 1 to 5 representing "totally disagree" to "totally agree" respectively integrating sixteen evaluation metrics over seven segments (see Table 1).

Table 1. Evaluation metrics and dimensions

\begin{tabular}{|c|c|c|}
\hline Dimensions & $\begin{array}{l}\text { Evaluation metrics and } \\
\text { ("n" no. of questions) }\end{array}$ & Details \\
\hline \multirow[t]{4}{*}{$\begin{array}{l}\text { Systems } \\
\text { quality }\end{array}$} & Adaptability 1 & $\begin{array}{l}\text { Information from various sources can be compared and consolidated } \\
\text { without more inconsistencies. }\end{array}$ \\
\hline & Reliability 1 & The consistency and dependability of the accessibility and system uptime. \\
\hline & Availability 6 & $\begin{array}{l}\text { Accessibility to computer infrastructure and resources when and where it } \\
\text { is required. }\end{array}$ \\
\hline & Usability 6 & $\begin{array}{l}\text { The capacity of human functionality terminologies to be applied } \\
\text { effectively and easily by various individuals, rendered particular training } \\
\text { and support, to accomplish a particular objective, within a particular } \\
\text { ecological setting. }\end{array}$ \\
\hline \multirow[t]{5}{*}{ Data quality } & Security 1 & $\begin{array}{l}\text { Safeguarding information from unauthorized alterations and } \\
\text { misappropriation or losses. }\end{array}$ \\
\hline & Ease-of-understanding 3 & $\begin{array}{l}\text { Easy determination of what information elements on reporting files imply } \\
\text { or what are integrated in evaluating it. }\end{array}$ \\
\hline & Completeness 2 & The understanding aspect of data contents. \\
\hline & Customization 1 & Capacity to customize HIS for individuals. \\
\hline & Relevance 1 & $\begin{array}{l}\text { The dimension of congruence between the needs of users and what avails } \\
\text { data services and products. }\end{array}$ \\
\hline \multirow{3}{*}{$\begin{array}{l}\text { Quality of } \\
\text { service }\end{array}$} & Responsiveness 4 & Willingness to assist clients and render the required services. \\
\hline & Assurance 4 & $\begin{array}{l}\text { Courtesy and knowledge of workers and their capacity to motivate } \\
\text { confidence and trust. }\end{array}$ \\
\hline & Empathy & Empathy caring personalized attention the providers of services provide. \\
\hline Fulfillment & $\begin{array}{l}\text { Assessment metrics } \\
\text { dimensionality } 4\end{array}$ & The dimension of general users' fulfillment with HISs. \\
\hline System use & $\begin{array}{l}\text { Assessment metrics } \\
\text { equal to dimensionality } \\
1\end{array}$ & The hours that individuals utilize HISs. \\
\hline Applicability & $\begin{array}{l}\text { Assessment metrics } \\
\text { equal to dimensionality } \\
\qquad 4\end{array}$ & $\begin{array}{l}\text { The dimension to which individuals believe that utilizing a novel scheme } \\
\text { would enhance the performance of work }\end{array}$ \\
\hline $\begin{array}{l}\text { Positive } \\
\text { externalities }\end{array}$ & $\begin{array}{l}\text { Assessment metrics } \\
\text { equal to dimensionality } \\
18\end{array}$ & $\begin{array}{l}\text { As the implication of HISs have transformed beyond the immediate } \\
\text { individuals, experts recommend more IS metrics e.g. workgroup affects, } \\
\text { inter-firm, industry effects, consumer effects and community effects. }\end{array}$ \\
\hline
\end{tabular}

\section{Research Settings}

The research was carried out at the mental clinic and teaching center of Dr. Hejazi in Ebnesina. The case clinic was a 900bed training clinic that had been open for 40 decades. The facility is Northeastern Persia's biggest mental facility. Individuals are transferred to this emergency clinic for specialist psychiatric therapy from five neighboring regions (with a demographic of around eleven million). Psychiatry emergency, paediatric psychiatric, geriatric psychiatry, substance addiction therapy, and adult psychiatry are only few of the 16 specialized and sub-specialized sections of the clinic. The mean bed utilization is 1.01, and the bed inventory turnover is $75 \%$. In general, people spend 9 days in the hospital. Mashhad University of Medical Technology launched the Iranian Health Information Systems (IHISs), a bespoke HIS, in 2002. IHISs are now applied in all the schools' clinics. Admissions, release, and transport, outpatient and inpatient, pharmacology, lab, radiography, accountancy, and insurance are all covered by the case clinic's IHIS.

\section{Participants}

Customers in four areas were surveyed: mental, administration and economic, nursing supervision, and para-hospitals Provided the massive number of healthcare practitioners operating in mental hospitals (n equals to 190), randomized sample of 64 healthcare practitioners was estimated. A total of 71 people from the psychiatry section took part in the survey, including 7 secretaries. All customers in other sectors (financial and administrative, nursing supervision, and paraclinics) were requested to engage in the present research, and a total of 61 people accepted. As a result, the research included a total of 125 respondents. Experimental data was gathered from users having at least 6 months of HIS expertise. To safeguard the respondents' privacy and rights, the research was authorized by Mashhad University ethics panel before instruments had formally provided all clients. Users were personally approached by the researchers, who encouraged them to partake in the research. Users who consented to take part in the research were given questionnaires. 


\section{Statistics}

Frequency and percentages were used to produce statistical results for individual attributes. The unfavorable replies were flipped on their heads. In the example clinic, the HIS effectiveness ratio was determined in two phases. Firstly, the percentage of HIS accomplishment by each user was established in terms of evaluation methods, aspects, and overall dimensions of the instruments. The following equations were used to do this:

- Ultimate HISs rate of success of assessment metrics, dimension and overall dimensions by every users equals to the overall questions $x 5$ (ultimate scores for every question on the Likert scale (1 to 5 point).

- The acquired success rate of HISs of assessment measure, dimension and overall dimensionality by every individual equals to the overall sum of the obtained scores for every question on the Likert scale (1 to 5 point) by every individual.

- The success rate of HISs equal to the obtained rate of success of HISs of assessing the metrics, dimensionality, and the overall dimension by every individual/maximum HIS rate of success of assessing measures, dimensionality and overall dimension by every user x 100 .

In the subsequent paragraphs, the calculations will be illustrated. The assessment metrics of "availability" belong to the quality of system dimensionality. This assessment metrics integrate six questions, and individuals reacted to every question based on the Likert scale ( 1 to 5 point). Maximized potential score for availability based on every individual was the same as a single individual x 6 questions x 5 score for every question onto the Likert scale ( 1 to 5 point) equals to 30 . HISs rate of success for the aspect of "availability" by the first user was 27. In that regard, HISs rate of success for "availability" by the first user equals to 27 out of $30 \times 100=90 \%$.

The "system quality" dimension integrates 14 questions. The ultimate potential score of the systems quality by every individual was the same as a single user x 14 questions x 5 ultimate score for every question equals to 70. HISs' rate of success of the system's quality by a single user was 51. In that regard, the success rate of HIS for the quality of system by a single user was 51 out of $71 \times 100=73 \%$.

The overall dimensionality of the instruments integrated of 61 questions. In that regard, the ultimate potential dimensionality score by every user was the same as one user x 60 questions x 5 maximum scores for every question equals to 300. The rate of success of HISs for the overall dimension of instruments by the firm user $=205$. In that regard, the success rate of HISs for the overall dimensions of instruments by the first user equals to 205 out of $300 \times 100=86 \%$.

In the $2^{\text {nd }}$ stage, four sets of customers calculated the average and credible range of the HIS effectiveness percentage for assessment methods, measurements, and overall proportions of the tool. Para-clinics sections, financial and administrative divisions, healthcare management divisions, and mental organizations are examples of these types of agencies. Many analytical procedures need a determination of information normalcy. As a result, the regularity of information in each ISSM category was analyzed in order to evaluate the HIS successfulness across four user groups. The Shapiro-Wilk test has been recommended by several academics as the best alternative for determining data normality. The Shapiro-Wilk assessment was applied in determination of consistencies of information in our research. The present research used ANOVA for standard parameters and the Kruskal-Wallis analysis for non-normal factors to compare the HIS successfulness across four customer segments. Statistical relevance was illustrated by the p-value significantly lesser that 0.1 .

In this research, two distinct post-hoc tests were selected in the process of identifying the intergroup variation at $p \geq$ 0.1. The HSD (Honesty Significant Difference) test for standard variable and Mann Whitney tests for the non-standard variable were considered. The analysis of data was done using the statistical software v. 11.5 of SPSS. Section III focuses on an analysis of experimentation results.

\section{RESULTS}

\section{Participants}

The process of the research included 191 intended audiences for this research, with 125 of them agreeing to take part. The features of the individuals are shown in Table 2. Women made up more than two-thirds of those who took part. Users ranged in age from 22 to 55, with the majority of them being in their 20s and 30s. $2 \%$ of those who took part lacked a college diploma. A two- or four-year bachelor's degree was held by greater than $77 \%$ of the participants. $61 \%$ of those who took part had worked for 5 to 15 years. The ICDL certificate was held by $68 \%$ of the users. Eighty percent of the customers had worked with HIS for more than three years, and sixty-six percent had worked with computers for more than six decades.

Table 2. Characteristics of participants

Participants' characteristics Sex

Age
Sub-segments

\begin{tabular}{cc} 
Males & $48 \%$ \\
Females & $77 \%$ \\
$20-30$ & $20 \%$ \\
$30-40$ & $73 \%$ \\
$40-50$ & $30 \%$ \\
Above 50 & $2 \%$ \\
High school diploma & $2 \%$ \\
\hline
\end{tabular}




\begin{tabular}{ccc}
\hline & Associate's degree & $2 \%$ \\
& Bachelor's degree & $94 \%$ \\
Monthly working hours & Master's degree & $27 \%$ \\
& Below regular + 20 hours overtime & $41 \%$ \\
& Regular + 20 to 60 hours overtime & $44 \%$ \\
& Regular + 20 to 120 hours & $30 \%$ \\
& overtime & $10 \%$ \\
Years of Experience & Above regular + 120 hours & $23 \%$ \\
& overtime & $35 \%$ \\
& Below 5 & $41 \%$ \\
& $5-10$ & $13 \%$ \\
Certification by ICDL & $10-15$ & $9 \%$ \\
& $15-20$ & $4 \%$ \\
Years of computing experience & $25-25$ & $97 \%$ \\
& Above 25 & $28 \%$ \\
\hline & Yes & $15 \%$ \\
\hline & No & $9 \%$ \\
\hline
\end{tabular}

Validity and Reliability

With a CVI of 0.85 and a CVR of 0.86 , the instrument was developed by an advisory group. The instrument's total Cronbach's alpha rating was 0.93 , indicating great dependability. The values ranged from 0.476 to 0.943 for various instrument diameters. Across the five categories of technology acceptance, quality of information, customer satisfaction, usability, and overall benefits, Cronbach's alpha was adequate. Cronbach's alpha for the contentment aspect was 47.0. Cronbach's alpha was not computed for the aspect "system utilization" since it only contained one question. The Pearson association value revealed substantial positive correlations between the variables studied (p-value: 0.01). The Pearson association value ranged from 0.197 to 0.707 for various dimensions.

\section{HISs' Success Rate}

The variables of "utility," "network efficiency "and" overall benefits" all had the greatest mean chances of success. The aspects of "system usage," "system integrity," and "data efficiency" were placed fourth through sixth, respectively.. In the next paragraphs, these findings will be examined in more detail.

\section{System Quality HISs' Success Rate}

Four assessment indicators were considered in the "systems efficiency" component, with an overall average positive outcome of 0.67 (95 percent CI: 65 percent, 69 percent) from the users' viewpoint. The average achievement level of assessment indicators in the "mechanism value" aspect is shown in Fig. 1. The overall performance of "flexibility" and "consistency" evaluation metrics was less than 60\%. Furthermore, assessment metrics of "accessibility" and "functionality" had a successfulness of more than $60 \%$.

This research found a substantial disparity in the average rate of success in the "accessibility" assessment metric among para-clinical and commercial and administration sectors ( $\mathrm{p}$ 0.001). In psychiatric facilities, there was a substantial variation ( $\mathrm{p}$ 0.001). In the finance and administration sections, healthcare management agencies, para-clinical divisions, and psychiatric agencies, the mean "accessibility" was 88 percent, 77 percent, 72 percent, and 68 percent, accordingly.

The study's findings revealed that the ICDL certification and the "accessibility" assessment metric differed significantly (p-value: 0.037). The degree of education has a substantial impact on the "usefulness" rating score. The average achievement for the "usability" assessment measure was significantly different between individuals with a qualification and those with a bachelor's degree ( $p$-value is 0.033 ). The disparity was visualized among the clients with master's degree and bachelor's degrees ( $p$-value is 0.033 ). The mean achievement for consumers with a qualification, bachelor's degree, and master's degree on this assessment measure was 78 percent, 66 percent, and 64 percent, accordingly.

The symbols "a" and "b" show substantial findings within every variety of consumers; values that do not share a similar letter vary substantially (P 0.05). 

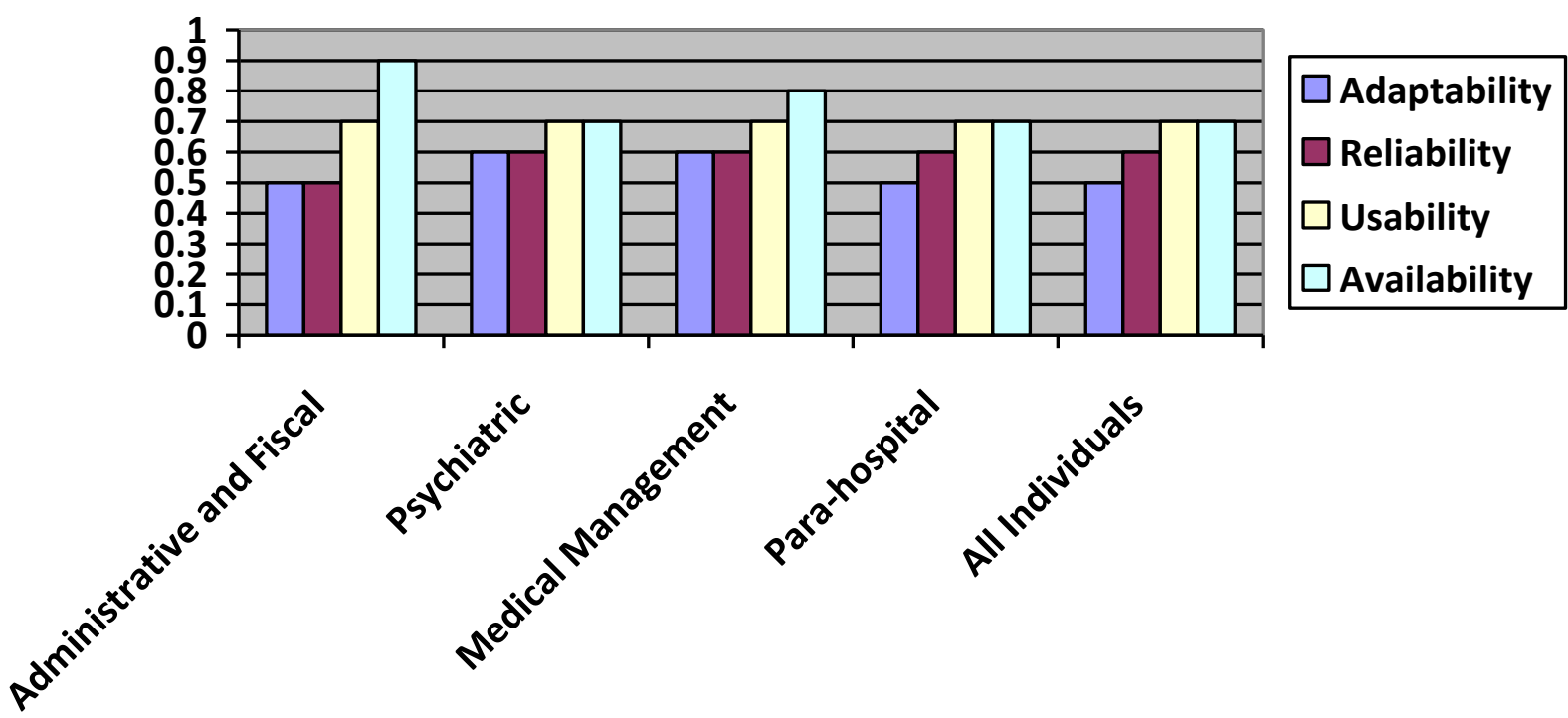

Fig 1. Average rate of success on the dimensionality of the system's quality

Rate of Success of HIS in Information Systems

Five variables make up the "data content" component, with an average rate of success of 0.63 (95 percent, CI: 61 percent, 64 percent) on users' perspectives. On the assessment metrics in this aspect, the average achievement ranged from $0.42-$ 0.73. "HIS privatization" and "HIS's information relevance," correspondingly, have the lowest and highest success percentages. The average achievement for this dimension's assessment measures and users' attributes did not vary significantly.

Rate of Success of HIS in Service Systems

Three assessment metrics make up the "customer value" component. Users gave this aspect 0.37 percent ( 95 percent CI: 61 percent, 65 percent) of the average rate of success (Fig. 3). There was no discernible variation in the average rate of success between assessment measures of this aspect and user attributes or groups of customers.

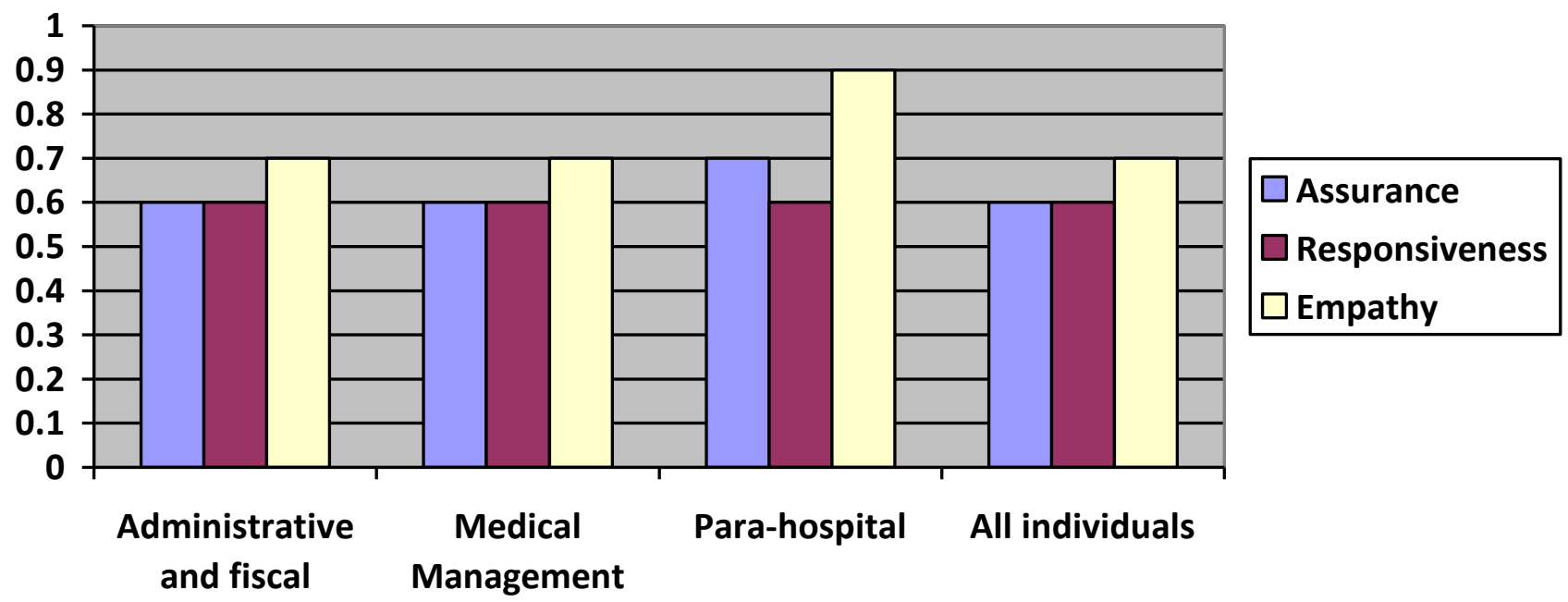

Fig 2. Average success rate on the dimensionality of the service quality

\section{DISCUSSION}

An ISSM-based device $[4,5]$ was created to analyze HISs in the present investigation. The reliability and validity of the tool were found to be quite good. In future investigations, this method might be used to assess different HISs. In addition, a mechanism for calculating the HIS rate of success depending on user feedback was devised. This technique allows consumers to compare the successfulness of HIS at different institutions. The researchers looked at the relationship between the average positive outcome of assessment aspects and user attributes such like age, gender, and user groups. The 
current researcher's findings shed light on the features and perspectives of HIS consumers in an improving nation. The survey's most significant results will be addressed in the preceeding sections.

ISSM has been updated and verified in [6] in health institutions. The current research verifies an ISSM-based survey in the settings of medical data networks at a developing nation training center. The present research found that the seven ISSM aspects, which comprise systems quality, data quality, quality of service, system use, applicability, fulfillment, and positive externalities, had a substantial link.

The findings in [7] indicated that consumers think HIS' achievement in the incident clinic is a good thing. Generally, the HIS rate of success was satisfactory in terms of usefulness, data security, accuracy, and work relevance. The majority of users looked at quality dimensions, system usage, and contentment. EHRs, according to [8], are capable of enhancing data quality and process. They also discovered that EHRs allow nurses to conveniently input, access, and interpret data, allowing them to do their tasks more quickly. Users perceive that EMR is straightforward to use and matched with their job, according to a research by [9]. Whenever EMRs are easy to utilize and integrated with daily routine, it is believable that the reliability of patient information improves. The outcomes of a research on EHR appraisal by [10] yielded comparable results. Overall, users were pleased with EHR's operational dependency, feedback speed, logins and assistances. In addition, EHR's performances were satisfactory. In the usefulness assessment measure, we found an acceptable average success rate. In our research, however, we discovered a substantial correlation between usability and educational level. Usability was perceived differently by consumers with a high school diploma, master's degree and bachelor's degree. According to the findings of their research, educational qualification has a substantial impact on reported simplicity of use and usefulness [12]. In 2021, a research [12] on the usability of IHIS was undertaken, and usability issues were discovered. These aesthetic issues in IHIS seem to have impacted users' judgments of usability at various levels of learning.

We discovered that there is indeed a considerable disparity between customer segments and the accessibility of computational facilities in this research. In comparison to the other three divisions, computer systems are more readily accessible in the administrative and financial sectors. Meanwhile, there was a considerable variation in system utilization and community groups. When compared to other users, people in the finance and administration sectors utilize HIS more often. Users with greater computer resources used HIS at a higher rate than other users.

\section{CONCLUSION}

In conclusion, Persia's data technologies are primarily centered on commercial goals. The average system utilization between customers of the healthcare department manager and psychiatric sections was found to be poor, according to our results. The results provided in this research are consistent with these observations. Because administrators and legislators are concentrated on HIS's commercial goals, there may be a considerable disparity in the pace of system usage, accessibility of computational resources, and organizational units. In comparison to users of psychiatric sections, nursing supervisors had greater access to network resources, according to the results of the present research. Nevertheless, there was no substantial difference in HIS utilization rates between the two categories. Furthermore, this research revealed that the organizational status of nurses had no effect on the frequency of HIS usage. In this investigation, we encountered a few constraints. Users' self-report was used to assess system utilization, which is a crucial metric in HIS assessment in this research. This might lead to an arbitrary assessment of the metric. Another issue was because the number of nodes in the psychiatry sections was inadequate, with most of them operating at low speeds. As a consequence, it was common for one user to $\log$ into HIS and for additional users to access the HIS using the first user's account. As a result, depending on HIS $\log$ data, we had been unable to appropriately reflect the exact level of system utilization. Systems quality, data quality, quality of service, system use, applicability, fulfillment, and positive externalities are among the seven aspects covered by this tool. Researchers may use the suggested approach to calculate the HIS successfulness based on these characteristics. The rate of success of HIS in each parameter is a numerical statistic. As a result, researchers now have an objective criterion to evaluate HIS rates of success across a broad variety of medical settings for future investigations. Our results also highlight the features and perspectives of HIS consumers in an emerging nation. According to our findings, HIS in the study hospital had a satisfactory successfulness (69\%) depending on user feedback. It's worth noting that various characteristics, such as usability, need to be tweaked and improved.

\section{References}

[1]. M. N. Ngafeeson and J. A. Manga, "The nature and role of perceived threats in user resistance to healthcare information technology: A psychological reactance theory perspective,” Int. J. Healthc. Inf. Syst. Inform., vol. 16, no. 3, pp. 21-45, 2021.

[2]. W. H. DeLone and E. R. McLean, “Information systems success measurement," Found. trends ${ }^{\circledR}$ inf. syst., vol. 2, no. 1, pp. 1-116, 2016.

[3]. H. Xiaoyang, Z. Junzhi, F. Jingyuan, and Z. Xiuxia, "Effectiveness of ideological and political education reform in universities based on data mining artificial intelligence technology,” J. Intell. Fuzzy Syst., vol. 40, no. 2, pp. 3743-3754, 2021.

[4]. L. A. Danso, J. K. Adjei, and W. Yaokumah, "EMIS success modeling using information systems quality factors," Int. j. inf. syst. serv. sect., vol. 13, no. 3, pp. 65-81, 2021.

[5]. K. Çelik and A. Ayaz, "Validation of the Delone and McLean information systems success model: a study on student information system," Educ. Inf. Technol., 2021.

[6]. I. A. Alfaki, "DeLone and McLean information systems success model in a blended-learning context," Int. J. Inf. Commun. Technol. Educ., vol. 17 , no. 4 , pp. $1-17,2021$.

[7]. "DeLone and McLean Information Systems Success Model in A Blended-Learning Context: D\&M ISS model in blended learning context," Int. J. Inf. Commun. Technol. Educ., vol. 17, no. 4, pp. 0-0, 2021. 
[8]. Y. C. Choi, D. Zhang, and J. E. Tyczynski, "Pcn338 metastatic non-small cell lung cancer (nsclc) patient characteristics and treatment patterns: A comparison between health insurance claims and electronic health records (EHRs)," Value Health, vol. 23, p. S84, 2020.

[9]. A. K. Barrett and K. K. Stephens, "Making electronic health records (EHRs) work: Informal talk and workarounds in healthcare organizations," Health Commun., vol. 32, no. 8, pp. 1004-1013, 2017.

[10]. S. Khairat, E. Metwally, C. Coleman, E. James, S. Eaker, and T. Bice, "Association between ICU interruptions and physicians trainees' electronic health records efficiency,” Inform. Health Soc. Care, vol. 46, no. 3, pp. 263-272, 2021.

[11]. Nishizono M., "Role, educational training and qualification of future psychiatrists--psychiatric specialists and physicians designated by mental health services," Seishin Shinkeigaku Zasshi, vol. 90, no. 11, pp. 1006-1014, 1988.

[12]. M. Mahdavi and H. S. Sajadi, "Qualitative analysis of Iranian sixth five-year economic, social, and cultural development plan from universal health coverage perspective," BMC Health Serv. Res., vol. 21, no. 1, p. 966, 2021. 